\title{
La corioamnionitis como hallazgo histopatológico. Instituto Materno Infantil
}

\author{
Jorge Andrés Rubio Romero*; Lilia María Sánchez**; Edith Angel***
}

\begin{abstract}
RESUMEN: La corioamnionitis es un hallazgo histológico frecuente. Sin embargo, el significado de este hallazgo no está bien aclarado, tanto desde el punto de vista histológico como clínico. Se realizó un estudio retrospectivo con el fin de describir las características generales de los casos con diagnóstico histopatológico de corioamnionitis encontrados en el Departamento de Patología del Instituto Materno Infantil y atendidos en esa institución durante el período de Junio 1 de 1994 a Mayo 31 de 1995. Se revisaron los antecedentes, hallazgos clínicos, mortalidad y hallazgos anatomopatológicos de los 104 casos revisados. La severidad de los hallazgos histológicos se relacionan con el antecedente de ruptura de membranas, el tiempo de esta y el diagnóstico clínico de corioamnionitis.
\end{abstract}

PALABRAS CLAVES: Corioamnionitis, histopatología, Mortalidad fetal, Ruptura prematura de Membranas.

SUMMARY: Chorioamnionitis is a common finding in histopathologic studies. However, clinical and pathologic significance of this findings are not very well defined. We review 104 cases of chorioamnionitis found in the Department of Pathology of the Instituto Materno Infantil (a terciary attention centre) in Bogotá, since June 11994 until May 31 1995. Obstetrical history, clinical findings, mortality and anatomo-pathologic findings were reviewed. Severity of the pathologic findings are related with premature rupture of the membranes, time of rupture and clinical diagnosis of chorioamnionitis.

KEY WORDS: Chorioamionitis, histopathology, fetal mortality, premature rupture of membranes.

\section{Introducción}

Los procesos inflamatorios de la placenta son un hallazgo histológico muy frecuente. Sin embargo, queda mucho por aprender sobre su naturaleza y significado; se mantienen varias controversias sobre: 1 . Los criterios diagnósticos para lesiones específicas. 2. La etiología y la patogénesis de la inflamación en la placenta, especialmente en relación con la infección y 3 . La relevancia clínica de tales hallazgos.

Los procesos inflamatorios en la placenta se diferencian por el patrón de compromiso. La corioamnionitis es el más conocido y se produce por infecciones ascendentes las cuales ocasionan una respuesta inflamatoria en las membranas fetales y el cordón llegando incluso a comprometer al feto. Es menos frecuente encontrar procèsos inflamatorios crónicos, que comprometen las vellosidades más que las membranas fetales. Este último patrón, la villitis, se observa en los procesos infecciosos que alcanzan la placenta a través de la sangre materna, siendo este proceso indicativo de enfermedad materna sistémica.

\footnotetext{
* $\quad$ Residente III Ginecología y Obstetricia Universidad Nacional de Colombia

** Instructora Asociada. Departamento de Patología. Universidad Nacional de Colombia.

*** Profesora Asistente. Departamento de Ginecología y Obstetricia.
} Universidad Nacional de Colombia.
La corioamnionitis es un hallazgo frecuente; Fox y Langeley la encontraron en 24,4 por mil nacimientos consecutivos (4), Solofi en el $4 \%$ de los nacimientos normales a término y Hillier en el $67 \%$ de los nacimientos prematuros $(5,6)$. Guzack y Winn la encontraron en el $11 \%$ de los partos prematuros de 2.774 nacimientos consecutivos, porcentaje que aumentó cuando se asoció a ruptura prematura de membranas al $56 \%$, lo que les permitió concluir que por lo menos el $25 \%$ de estos partos eran atribuibles a corioamnionitis (7).

La corioamnionitis es más grave cuando se presenta durante los dos primeros trimestres del embarazo debido a factores asociados a la deficiencia inmunológica del feto dada por la inmadurez, con una alta tasa de mortalidad fetal. Hacia el final de la gestación, la frecuencia de la corioamnionitis está relacionada con la paridad, la sobredistensión uterina y a la pérdida del moco cervical que expone la superficie de las membranas a la flora vaginal y cervical (8). La presentación de corioamnionitis también se ve favorecida por el coito, la monitorización fetal interna, el trabajo de parto, la multiparidad, la edad gestacional, el antecedente de corioamnionitis, la incompetencia cervical y la instrumentación del cuello (aborto inducido, cerclaje, etc). Se ha encontrado con mayor frecuencia en las poblaciones de bajos recursos económicos, en la raza negra y en las pacientes desnutridas.

Ocasionalmente, la corioamnionitis se inicia luego de procedimientos de diagnóstico obstétrico como 
amniocéntesis, fetoscopia, biopsia de las .vellosidades coriales o cualquier otro procedimiento que exponga las membranas fetales al medio externo.

En los estudios en los que se han cultivado las placentas, se ha logrado demostrar que la etiología de la corioamnionitis es bacteriana o por Mycoplasmas $(M$. hominis y U. urealyticum) (10). Al parecer por la presencia de sustancias antimicrobianas al final de la gestación en el líquido amniótico, es difícil aislar gérmenes a este nivel (13), aunque en presencia de manifestaciones clínicas de corioamnionitis se reportan cultivos positivos en líquido amniótico hasta en el $95.1 \%$ de los casos (10).

El hallazgo de $C$. trachomatis y $G$. vaginalis en el cervix parece predisponer a la aparición de otros factores asociados a la infección corioamniótica a pesar de no haber sido encontrados como agentes causales en algunos estudios. La tabla 1 resume los organismos aislados en cultivo tanto de líquido amniótico como de las membranas fetales (10).

Tabla 1

\section{Organismos}

\section{Aerobios}

Gram Positivos

Lactobacilos

S. epidermidis

Streptococo Grupo B

Enterococos

S. alpha hemolítico

S. aureus

Difteroides

Otros

Bacilos Gram Negativos

Klebsiella pneumonie

Gardnerella vaginalis

Escherichia coli

Proteus mirabilis

Enterobacter aerogenes

\section{Anaerobios}

Cocos Gram positivos

Peptostreptococos sp

Peptococos sp

Bacilos Gram positivos

C. perfringes

Clostridia sp

Eubacterium $\mathrm{sp}$

Otros

Bacilos gram negativos

Bacteroides bivius

Fusobacterium sp

Bacteroides fragilis

\section{Otros}

Cándida álbicans

Ureaplasma urealyticum

Mycoplasma hominis
La histopatología de la corioamnionitis ha sido descrita desde 1929. Se trata de una respuesta inflamatoria aguda con participación principalmente de polimorfonucleares neutrófilos y eosinófilos; cuando el curso es prolongado también pueden verse macrófagos. El infiltrado inflamatorio linfoplasmocitario es raro. Antes de la semana 20 de gestación los neutrófilos son de origen materno; posteriormente el feto adquiere la capacidad de generar factores quimiotácticos y activadores, que promueven la migración de neutrófilos fetales. Dichas células tienen capacidad fagocítica reducida aún hacia el final de la gestación, haciendo que el feto sea susceptible a la sepsis (13).

Microscópicamente, puede dividirse en tres estadios la respuesta inflamatoria en las membranas (16):

Estadio 1: Los neutrófilos se fijan a la placa coriónica a partir del espacio intervelloso y la decidua capsularis. Este estadio dura en promedio tres días.

Estadio 2: Permeación de la placa coriónica por los leucocitos.

Estadio 3: Los neutrófilos alcanzan la membrana basal del amnios (corioamnionitis). La duración media es de 7 días.

La severidad de la corioamnionitis puede clasificarse según la cantidad de neutrófilos que infiltran las membranas (3):

-leve (+) en la que, a gran aumento, solo se observan de 1-3 neutrófilos por campo.

-moderada (++) si se observan de 4-15 neutrófilos por campo.

-severa (+++) si se observan más de 15.

En el cordón, la migración de los neutrófilos comienza en la vena umbilical y luego en las arterias umbilicales hacia la cavidad amniótica. El hallazgo de funisitis no implica compromiso o sepsis del feto, aunque si parece tener relación con la presencia de calcificaciones o trombosis del cordón (3). Sin embargo, este hallazgo ha sido correlacionado con infecciones de mayor severidad por compromiso inflamatorio coincidente de las membranas y el plato corial, y mayor recuento de colonias bacterianas en el líquido amniótico (10).

La poca permeación de las células inflamatorias hacia la cavidad amniótica se debe a la presencia de colágeno tipo $\mathrm{V}$ en la membrana basal del epitelio amniótico. Los neutrófilos carecen, al parecer, de colagenasas específicas para este tipo de proteína (17). A pesar de esto, puede encontrarse pus en el oído medio, el estómago y el pulmón de recién nacidos cuyo embarazo ha cursado con corioamnionitis. En 1962 Mac Lelan encontró correlación ente la presencia de otitis media y la historia de corioamnionitis (3).

La corioamnionitis se manifiesta clínicamente con la aparición de fiebre materna, taquicardia, leucocitosis, sensibilidad uterina, descarga purulenta o de olor fétido por la vagina y compromiso fetal dado por taquicardia o en forma mas tardía y severa, bradicardia fetal. La alteración del perfil biofísico fetal es considerada por algunos autores otra manifestación clínica de compromiso fetal por infección intraamniótica $(27,31)$. Estos criterios son indicadores tardíos de infección y por este motivo, son necesarios una fuerte sospecha clínica y la 
Tabla 2

GRADO DE SEVERIDAD DE LA CORIOAMNIONITIS POR GRUPOS DE EDAD GESTACIONAL

\begin{tabular}{|c|c|c|c|c|}
\hline Edad Gestacional & $\begin{array}{l}\text { Menor de } 28 \\
\text { Semanas }\end{array}$ & $\begin{array}{l}\text { Entre } 28 \text { y } 37 \\
\text { Semanas }\end{array}$ & $\begin{array}{l}\text { Mayor de } 37 \\
\text { Semanas }\end{array}$ & Total \\
\hline \multicolumn{5}{|l|}{ Grado de Corioamnionitis } \\
\hline Leve & $14(24.6 \%)$ & $4(14.3 \%)$ & $4 \quad(21.0 \%)$ & $22(21.2 \%)$ \\
\hline Moderada & $2(3.5 \%)$ & $1(3.6 \%)$ & $1 \quad(5.3 \%)$ & $4 \quad(3.8 \%)$ \\
\hline Severa & $22(38.6 \%)$ & $10(35.7 \%)$ & $3(15.8 \%)$ & $35 \quad(33.7 \%)$ \\
\hline No Clasificada & $19(53.3 \%)$ & $13(46.4 \%)$ & $11(57.9 \%)$ & $43 \quad(41.3 \%)$ \\
\hline Total & $57(100 \%)$ & $28(100 \%)$ & $19(100 \%)$ & $104(100 \%)$ \\
\hline
\end{tabular}

realización de pruebas diagnósticas como el recuento leucocitario, determinación de estearasa leucocitaria, niveles de glucosa intraamniótica, interleucinas, prostaglandinas, leucotrienos y Gram y cultivo del líquido amniótico, y algunas pruebas en suero materno como proteína C reactiva y específicas para algunos microorganismos causales, incluida la reacción de polimerasa en cadena. Los altos costos y la dificultad técnica para la realización de estos exámenes dificultan en gran medida el diagnóstico precoz de esta entidad (28-29).

\section{Metodología}

Se revisaron los informes de patología del Departamento de Patología del Instituto Materno Infantil de la Ciudad de Santafé de Bogotá, seleccionando todos los casos con diagnóstico de Corioamnionitis Aguda o Funisitis como hallazgo histopatológico entre el 1 de Junio de 1994 y el 31 de Mayo de 1995, analizando las historias clínicas correspondientes.

La información fue recolectada en un formulario diseñado exclusivamente para tal fin, incluyendo las variables a estudiar, el número de Historia Clínica y el diagnóstico Histopatológico y de severidad de la corioamnionitis o funisitis.

La información obtenida fue analizada con el programa EPI INFO versión 6.0 para realizar análisis de estadística descriptivo y pruebas de significancia Chi cuadrado y t student donde fue apropiado.

\section{Resultados}

Realizada la búsqueda, se encontraron 109 casos en el período estudiado, de los cuales fue posible revisar 103 historias clínicas. Una paciente presentó dos pérdidas gestacionales durante el período estudiado, en ambas ocasiones con diagnóstico de corioamnionitis, para un total de 104 casos estudiados.

Se analizaron los casos de acuerdo con la edad gestacional estableciendo grupos de la siguiente forma: gestaciones menores de 28 semanas, gestaciones con parto pretérmino (edad gestacional entre 28 y 37 semanas) y embarazos a término, encontrando los siguientes hallazgos:

Clasificación histológica: Las corioamnionitis fueron clasificadas histopatológicamente solo en el 58.7\%(61 casos) de los informes patológicos, distribuidos de la siguiente forma: leve en el $21.2 \%$ ( 22 casos), moderada $3.8 \%$ (4 casos) y severa en el $33.7 \%$ (35 casos). El $41.3 \%$ de las corioamnionitis diagnosticadas por patología no fue clasificado. La tabla 2 y la figura 1 muestran la distribución por grupos.

Edad gestacional: La edad gestacional promedio de los casos revisados fue de 26.7 semanas, con un rango de 8 a 41 semanas. Sin embargo, el mayor número de corioamnionitis se diagnosticó en placentas de embarazos de 37 semanas, siendo 10 en total para un $9.6 \%$ de los casos.

El $29.8 \%$ de los casos ocurrió en gestaciones menores de 20 semanas, el $23.1 \%$ en gestaciones entre las 20 y 28 semanas y el $47.9 \%$ fue diagnosticado en placentas del último trimestre de embarazo.

Se encontró el diagnóstico de Funisitis en 25 casos (24\%), asociado a corioamnionitis severa en el $40 \%$ de ellos. En tres ocasiones se asoció el diagnóstico de abruptio de placenta con el de corioamnionitis, en dos con el de neumonía intrauterina fetal y un caso de anencefalia con corioamnionitis con infiltrado inflamatorio crónico.

Figura 1

\section{CLASIFICACION DE SEVERIDAD SEGUN EDAD GESTACIONAL}

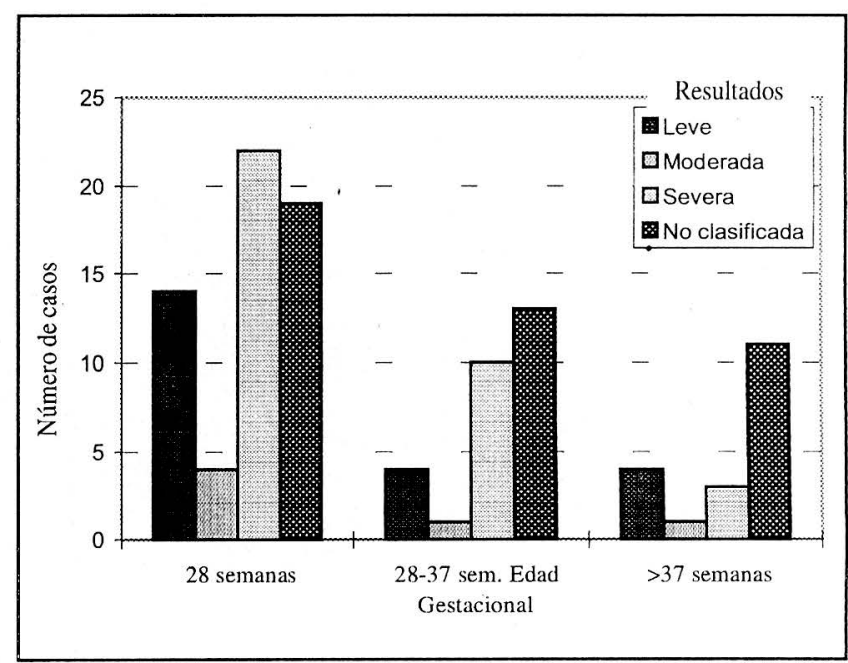


Tabla 3

DISTRIBUCION DE LA POBLACION POR GRUPOS DE EDAD

\begin{tabular}{|lcc|}
\hline Grupos de Edad & $\begin{array}{c}\text { Número de } \\
\text { Pacientes }\end{array}$ & Porcentaje \\
\hline 10-19 Años & 28 & $26.9 \%$ \\
20-29 Años & 51 & $49.0 \%$ \\
30-39 Años & 21 & $20.2 \%$ \\
40-49 Años & 4 & $3.8 \%$ \\
Total & 104 & $100 \%$ \\
\hline
\end{tabular}

Edad: El promedio de edad de las pacientes fue de 24.5 años con un rango de 13 a 41 años. La distribución por grupos de edad se muestra en la tabla 3.

Paridad: El $39.4 \%$ de las pacientes eran primigestantes, el $13.4 \%$ secundigestantes, $22.2 \%$ cursaban su tercera gestación y el $25 \%$ tenían más de tres gestaciones previas. El $21.2 \%$ de las pacientes tenían antecedente de abortos previos, y solo 2 pacientes $(1.9 \%)$ podrían considerarse abortadoras habituales.

Antecedente de Ruptura Prematura de Membranas: Del total de los casos, existió antecedente claro de ruptura prematura de membranas en $46(44.2 \%)$. En el $55.8 \%$ restante no se pudo establecer el antecedente dentro de la historia clínica (edad gestacional temprana, la paciente no refirió amniorrea ) o se encontró integridad de las membranas durante su estancia hospitalaria (Tabla 4). De los 46 casos con antecedente de ruptura de membranas, el $67.4 \%$ tuvo una latencia mayor de 12 horas al momento de terminación de embarazo (Figura 2).

Diagnóstico Clínico: Solo se sospechó el diagnóstico clínico de corioamnionitis en el $36.8 \%$ de todos los casos, bien por el antecedente de aborto provocado (en dos casos), ruptura prematura de membranas con fiebre materna (4 casos) y en la mayoría de los casos por los hallazgos durante la revisión uterina post parto o durante el acto quirúrgico de la cesárea por el hallazgo de líquido amniótico fétido o purulento.

Terminación de la Gestación: El $27.9 \%$ de las gestaciones terminó en legrado obstétrico (todas las gestaciones menores de 20 semanas), el $50 \%$ en parto
Figura 2

\section{ANTECEDENTE DE RPM Y TIEMPO DE RUPTURA POR EDAD GESTACIONAL}

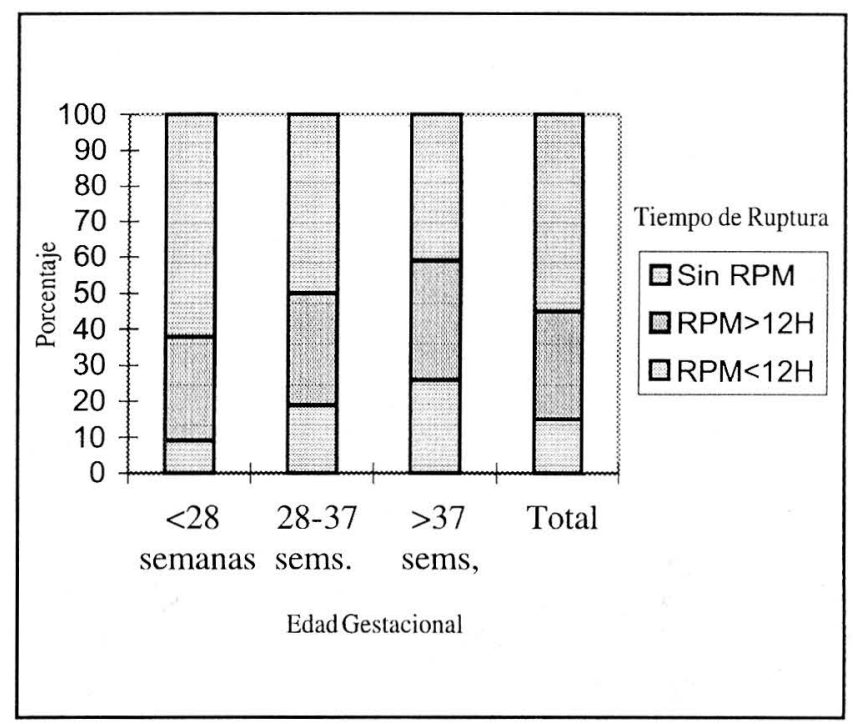

vaginal y el $22.1 \%$ en parto por operación cesárea. Se observó una mayor proporción de partos quirúrgicos en los embarazos a término que en los prematuros viables (Tabla 5).

Mortalidad: La mortalidad fetal en los casos estudiados fue de $63.3 \%$ (69 casos) con grandes variaciones según la edad gestacional. Por debajo de las 28 semanas la mortalidad fetal fue del $96.5 \%$, entre la semana 28 y la 37 el porcentaje de óbitos fetales fue del $43 \%$ y en embarazos a término fue del $10.5 \%$, incluyendo en este grupo otras causas que pudieron asociarse a la muerte fetal, como el abruptio placentario, sufrimiento fetal agudo, insuficiencia placentaria e hipertensión inducida por el embarazo. La Tabla 6 muestra la Mortalidad Fetal ( Figura 3).

En el grupo de menores de 28 semanas, hubo dos nacimientos vivos, que por su prematurez, fallecieron en la Unidad de cuidado intensivo neonatal menos de 12 horas después de su nacimiento. Se presentaron

Tabla 4

ANTECEDENTE DE RPM, Y TIEMPO DE RUPTURA POR GRUPOS DE EDAD GESTACIONAL

\begin{tabular}{|lllll|}
\hline Edad gestacional & $\begin{array}{l}\text { Menor de 28 } \\
\text { Semanas }\end{array}$ & $\begin{array}{c}\text { Entre 28 y 37 } \\
\text { Semanas }\end{array}$ & $\begin{array}{c}\text { Mayor de 37 } \\
\text { Semanas }\end{array}$ & Total \\
\hline $\begin{array}{l}\text { Antecedente de RPM } \\
\text { < de 12 Horas }\end{array}$ & $5(8.7 \%)$ & $5(17.9 \%)$ & $5(26.3 \%)$ & $15 \cdot(14.4 \%)$ \\
> de 12 Horas & $16(28.1 \%)$ & $9(32.1 \%)$ & $6(31.6 \%)$ & $31(29.8 \%)$ \\
Total RPM & $21(36.8 \%)$ & $14(50 \%)$ & $11(57.9 \%)$ & $46(44.2 \%)$ \\
Total casos & $57(100 \%)$ & $28(100 \%)$ & $19(100 \%)$ & $104(100 \%)$ \\
& & & & \\
RPM: Ruptura Prematura de Membranas.
\end{tabular}


Tabla 5

VIA DE TERMINACION DEL EMBARAZO POR GRUPOS DE EDAD GESTACIONAL

\begin{tabular}{|llllll|}
\hline Edad Gestacional & $\begin{array}{l}\text { Menor de 28 } \\
\text { Semanas }\end{array}$ & $\begin{array}{l}\text { Entre 28-37 } \\
\text { Semanas }\end{array}$ & $\begin{array}{l}\text { Mayor de 37 } \\
\text { Semanas }\end{array}$ & Total \\
\hline Via de Terminación & $29(50.9 \%)$ & $0 \quad(-\%)$ & 0 & $(-\%)$ & $29(27.9 \%)$ \\
Legrado & $26(45.6 \%)$ & $18(64.3 \%)$ & $8(42.1 \%)$ & $52(50.0 \%)$ \\
Parto Vaginal & $2(3.5 \%)$ & $10(35.7 \%)$ & $11(57.9 \%)$ & $23(22.1 \%)$ \\
Cesárea & $57(100 \%)$ & $28(100 \%)$ & $19(100 \%)$ & $104(100 \%)$ \\
Total & & & & & \\
\hline
\end{tabular}

Figura 3

MORTALIDAD FETAL SEGUN EDAD GESTACIONAL

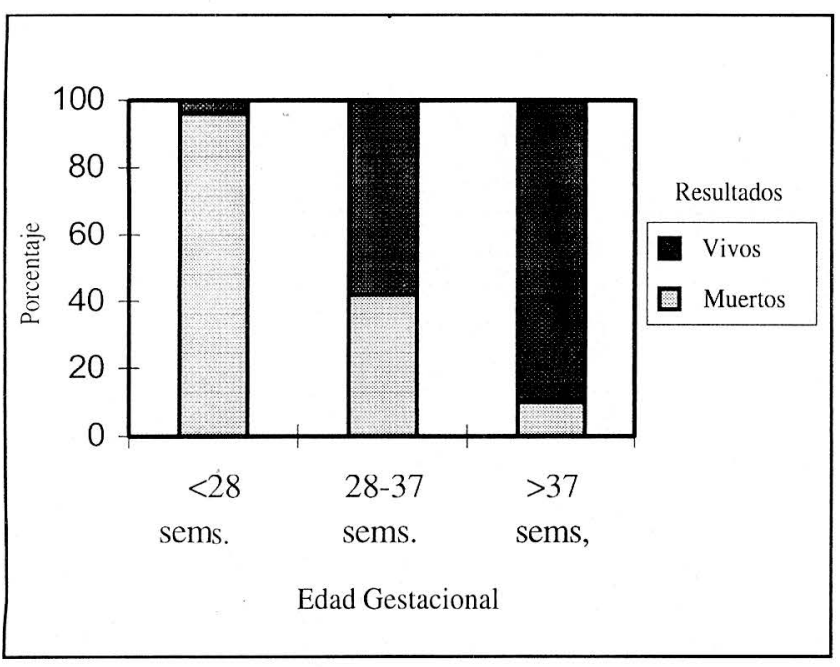

corioamnionitis leves en el $24.6 \%$ de dichas placentas, moderadas $3.5 \%$ y severas en el $38.6 \%$. El $33.3 \%$ de las corioamnionitis no fueron clasificadas según su grado de severidad. No fue posible establecer relaciones estadísticamente significativas entre el grado de severidad o la presencia de funisitis, el antecedente de ruptura y el tiempo de latencia de la misma con la mortalidad por la gran cantidad de placentas sin clasificar y la alta mortalidad presente en esta edad gestacional. En los 28 casos con feto viable pretérmino, se encontró corioamnionitis severa en el $35.7 \%, 3.6 \%$ moderada y $14.3 \%$ corioamnionitis leve. El $46.4 \%$ de los casos no fue clasificado. El antecedente de ruptura prematura de membranas estuvo presente en el 50\% de los casos, el $64.3 \%$ de ellos con latencia mayor de 12 horas. Sin embargo, no se encontró asociación estadísticamente significativa entre el grado de severidad o la presencia de funisitis con el tiempo de ruptura de las membranas o la mortalidad fetal, por las mismas razones anteriormente expuestas.

En las 19 gestaciones a término, se encontró corioamnionitis leve en el $21 \%$ de los casos, moderada en $5.3 \%$ y severa en el $15.8 \%$. Un alto porcentaje de las corioamnionitis para este grupo (57.9\%) no fue clasificado histopatológicamente.

El antecedente de ruptura de membranas estuvo presente en el $57.9 \%$ de los casos, con latencia mayor de 12 horas en el $54.5 \%$ de ellos. Tampoco en este grupo se encontró relación estadísticamente significativa entre los grados de severidad y la presencia de funisitis con el tiempo de ruptura o la mortalidad fetal.

Al analizar el diagnóstico clínico de la entidad se encontró que para todas las gestaciones el grado de severidad en la clasificación histopatológica fue mayor cuando se presentaron manifestaciones clínicas de corioamnionitis, según se muestra en la tabla 7 y la figura 4 , siendo este hallazgo significativo estadísticamente con un valor de $\mathrm{p}<0.05$.

\section{Discusión}

La corioamnionitis aguda es un hallazgo frecuente en los estudios histopatológicos de las placentas. Sin embargo, no es posible determinar la incidencia real de este hallazgo debido a que no se analizaron todas las placentas obtenidas durante el período estudiado sino solo aquellas con el diagnóstico específico. Hay que considerar, además, que todas las placentas obtenidas en el Instituto no

Tabla 6

MORTALIDAD FETAL POR GRUPOS DE EDAD GESTACIONAL

\begin{tabular}{|lllll|}
\hline Edad Gestacional & $\begin{array}{l}<\text { de 28 } \\
\text { Semanas }\end{array}$ & $\begin{array}{l}\text { Entre 28 y 37 } \\
\text { Semanas }\end{array}$ & $\begin{array}{l}\text { Mayor de 37 } \\
\text { Semanas }\end{array}$ & Total \\
\hline $\begin{array}{l}\text { Mortalidad } \\
\text { No. de Muertes fetales }\end{array}$ & $55(96.5 \%)$ & $12(42.9 \%)$ & $2(10.5 \%)$ & $69(63.3 \%)$ \\
Total de Casos & $57(100 \%)$ & $28(100 \%)$ & $19(100 \%)$ & $104(100 \%)$ \\
\hline
\end{tabular}


Tabla 7

DIAGNOSTICO CLINICO SEGUN GRADO DE SEVERIDAD HISTOLOGICA

\begin{tabular}{|lccc|}
\hline $\begin{array}{l}\text { Edad } \\
\text { Gestacional }\end{array}$ & $\begin{array}{c}\text { Menor de 28 } \\
\text { Semanas }\end{array}$ & $\begin{array}{c}\text { Entre 28 y 37 } \\
\text { Semanas }\end{array}$ & $\begin{array}{c}\text { Mayor de 37 } \\
\text { Semanas }\end{array}$ \\
\hline Dx Clínico & & & \\
Positivo & 15 & 11 & 26 \\
Negativo & 20 & 58 & 78 \\
Total & 35 & 69 & 104 \\
\hline
\end{tabular}

Nota: La categoría No Severo Incluye Leve, Moderada y No Clasificada.

\section{Figura 4 \\ DIAGNOSTICO CLINICO SEGUN GRADO DE SEVERIDAD HISTOLOGICA}

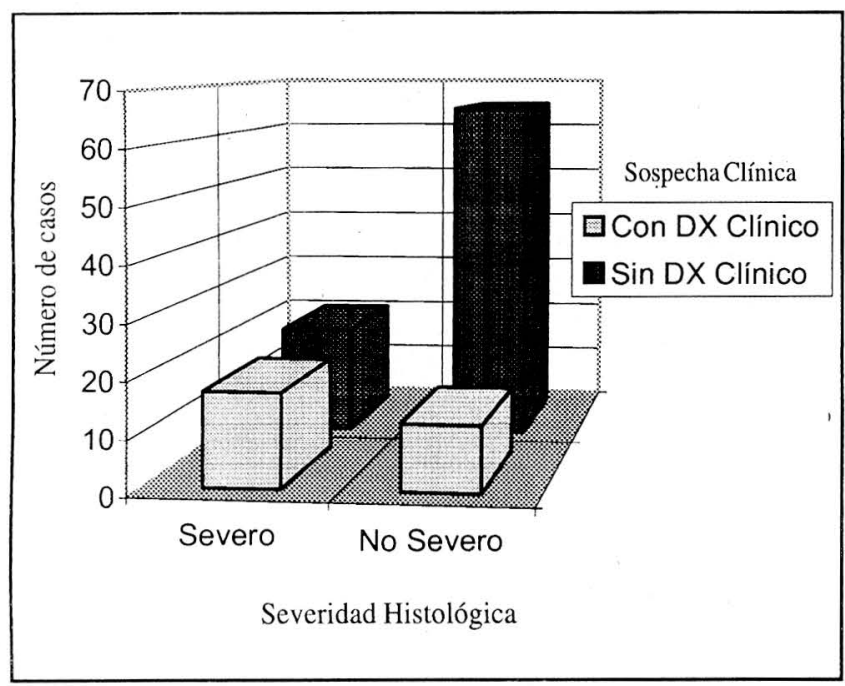

son enviadas a estudio anatomopatológico, sino solo aquellas que a criterio del obstetra lo ameritan.

La corioamnionitis afecta las mujeres a lo largo de toda su vida fértil, sin afectar ningún grupo etario en especial, como lo demuestra su amplia distribución desde los 13 hasta los 41 años.

Tal como lo describe la literatura (2), la mayor parte de las corioamnionitis se presentaron en mujeres multigestantes; el $47 \%$ se presentaron en mujeres con tres o más gestaciones y el $39.4 \%$ en primigestantes. El antecedente de abortos previos no parece influir en la aparición de corioamnionitis.

El antecedente de infección corioamniótica en gestaciones previas es factor de riesgo para la aparición de nuevas corioamnionitis $(2,11)$. En el estudio, solo una de las pacientes durante el período estudiado presentó dos pérdidas gestacionales con hallazgo de corioamnionitis en ambas ocasiones. El corto período analizado impide establecer la magnitud de esta asociación.
El hallazgo de inflamación de las membranas tuvo una amplia distribución en las diferentes edades gestacionales. Sin embargo, se encontró una mayor proporción en el último trimestre de la gestación, de acuerdo con lo descrito en la literatura (11).

La severidad de la inflamación de las membranas es difícil de correlacionar en el presente estudio debido a diversos factores. Los diferentes estudios publicados sobre corioamnionitis histopatológica utilizan diversos criterios diagnósticos para determinar el grado de inflamación, así, algunos utilizan la profundidad de la infiltración de los neutrófilos maternos dentro de las membranas (estadios 1-3) y otros el número de neutrófilos por campo de gran aumento $(2,10,19)$. La falta de criterios específicos determinó el alto porcentaje de reportes sin clasificar y la ausencia de descripción microscópica en los reportes de patología impidió el intento de clasificar en forma más uniforme la severidad de las corioamnionitis.

Del mismo modo, la presencia de funisitis es considerada por algunos autores signo de mayor severidad de la infección, relacionada con el compromiso de los vasos del cordón umbilical y el resultado fetal. En esta serie, se observó la tendencia de aparición de funisitis en los casos con mayor grado de severidad de inflamación de las membranas a pesar del alto número de corioamnionitis no clasificadas.

El inconveniente de la falta de clasificación de las corioamnionitis tampoco permite establecer con certeza la relación que pueden tener el antecedente de ruptura de membranas y el tiempo de latencia, con la mayor o menor respuesta inflamatoria de las membranas. Se ha encontrado que el grado de infiltración depende mas del tipo de microorganismo, su virulencia, y el recuento de colonias en las membranas y en el líquido amniótico. Igual sucede con el compromiso fetal y perinatal (10-12).

La alta mortalidad observada no se pudo correlacionar con el grado de inflamación de las membranas. Sin embargo, se observa la tendencia a encontrar mayor proporción de corioamnionitis severas en edades gestacionales más tempranas asociadas a mayor mortalidad fetal. Esto puede explicarse gracias a la capacidad reducida del feto de defenderse debido a su inmadurez inmunológica y a la constitución histológica de las membranas en etapas tempranas. Con el avance de la gestación, las membranas adquieren una consistencia firme y resistente, característica que pierden solo cuando se acerca el término del embarazo, lo que retrasaría la infiltración de las membranas en las gestaciones en el tercer trimestre $(1,3,17)$.

La resistencia de las membranas se ve disminuida por la acción de diferentes sustancias mediadoras de inflamación, así como por la acción directa de las bacterias que las colonizan, lo que favorecería la ruptura prematura de las membranas. Igualmente, las sustancias mediadoras de la inflamación liberadas por el corion, las membranas y la decidua, desencadenan el trabajo de parto (20-26), lo que se refleja en el hecho que el $26.9 \%$ de los casos analizados correspondió a placentas entre las 28 y las 37 semanas de los cuales el $50 \%$ tuvo antecedente de ruptura prematura de membranas. En las placentas a término, un porcentaje 
similar $(57.9 \%)$ tuvo antecedente de ruptura. No es posible establecer las causas del mayor número de partos quirúrgicos en los embarazos a término.

La sospecha clínica de corioamnionitis se relacionó con el grado de infiltración de las membranas tal como está informado en las diferentes publicaciones $(10,19)$, en las que se menciona que después del diagnóstico clínico, el siguiente factor relacionado con el grado de severidad de la inflamación de las membranas es el recuento de colonias en el líquido amniótico. Lo anterior, refleja la respuesta local que se genera con la infección de las membranas, y que a medida que avanza llega a comprometer a la madre y al feto generando una respuesta sistémica. Sin embargo, no se ha podido establecer con claridad la relación entre la infiltración de las membranas, la infección intraamniótica y la sepsis perinatal (30).
La prevención constituye un reto en el manejo de la corioamnionitis aguda, por la consiguiente morbi-mortalidad asociada tanto a la prematurez como a la infección neonatal. Es por esto que el control prenatal cuidadoso, la erradicación de la infección vaginal, la disminución de la actividad sexual o el uso de preservativos, la detección precoz de la dilatación cervical y el trabajo de parto y el empleo de antibióticos para el tratamiento de las infecciones cervico-vaginales y por que no, en la amenaza de parto pretérmino, pueden ser útiles en la prevención del parto pretérmino y de la ruptura prematura de membranas asociadas a la infección corioamniótica (30).

Se recomienda la clasificación rutinaria del grado de infiltración de las membranas corioamnióticas como un parámetro de la severidad de la infección, su evolución y sus posibles implicaciones perinatales.

\section{BIBLIOGRAFIA}

1. Kurman RJ. Blaunstein's Pathology of the female genital tract. Third ed. Springer-Verlag, New York. 1987.

2. Naeye RL. Placenta y membranas. En: Charles D. ed. Infecciones obstétricas y perinatales. Primera ed. Mosby/Doyma. 1994.

3. Benirschke K Infectious Diseases. En: Bernirschke K, eds. Pathology of the human placenta. 2 ed. Springer-Verlag, 1990.

4. Fox H. and Langley FA. Leucocitic infiltration of the placenta and umbilical cord: a clinicopathological study. Obstet Gynecol 1974; 37: $451-458$

5. Salafia CM, Silberman L, Herrera NE, Mahoney ML. Placental pathology at term associated with elevated midtrimester serum alpha feto protein concentration. Am J Obstet Ginecol 1988; 158: 10641066

6. Hillier SL, Martius J, Krohn M, Kiviat N, Holmes KK, Eschenbach DA. A case-control study of chorioamnionic infection and histologic chorioamnionitis in prematurity. N Eng J Med 1988; 319 : 972-978

7. Guzick DS and Winn K. The association of chorioamnionitis with preterm delivery. Obstet Gynecol 1985; 65: 11-16

8. Naeye RL and Peters EC. Causes and consecuences of rupture of fetal membranes. Lancet 1980; 1:192-194

9. Naeye RL and Ross SM. Amniotic fluid infection syndrome. Clin Obstet Gynecol 1982; 9: 593-607

10. Dong Y, St Clair PJ, Ramzy I, Kagan-Hallet KS, Gibbs RS. A microbiologic and clinical study of placental inflammation at term. Obstet Gynecol 1987; 70: 175-182

11. Naeye RL and Tafari N. Risk Factors in pregnancy and diseases of the fetus and newborn. Baltimore. Williams \& Wilkins. 1983.

12. Romero R, Sirtori M, Oyarzun E. et al. Prevalence, microbiology and clinical significance of intra amniotic infection in women with preterm labor and intact membranes. Am J Obstet Gynecol 1989;161: 817-824

13. Nazir MA, Pankuch GA, Botti JJ, Appelbaum PC. Antibacterial activity if amniotic fluid in the early third trimester. Am J Perinatol 1987; 4: 59-62

14. Vintzileos AM, Campbell WA, Nochimson DJ,Weinbaum PJ. Preterm premature rupture of membranes: a risk factor for the development of abruptio placentae. Am J Obstet Gynecol 1987; 156: 1235-1238

15. Darby MJ, Caritis SN, Shen-Schwartz S. Placental abruption in the preterm gestation: an association with chorioamnionitis. Obstet Gynecol 1989; 74:88-92

16. Blanc W. Pathology of the Placenta, membranes and umbilical cord in bacterial, fungal and viral infection in man. En: Naeye RL, Kissane
JM, Kaufman N, eds. Perinatal Diseases, Baltimore: Williams and Wilkins, 1981: 67-132.

17. Azarelli B and Lafuze J. Amniotic basement membrane: a barrier to neutrofil invasion. Am J Obstet Gynecol 1987; 156: 1130-1136

18. Naeye RL and Ross SM. Coitus and Chorioamnionitis: a prospective study. Early Hum Dev 1982; 6: 91-97

19. Naeye RL. Disorders of the placenta, fetus and neonate, their diagnosis and clinical significance. St Louis, Mosby YearBook, 1991.

20. Romero R, Emamian M, Quintero R, et al. Amniotic Fluid Prostaglandin levels and intraamniotic infections. Lancet 1986; 1: 1380.

21. Romero R, Kadar N, Hobbins JC, Duff GW. Infection and labor: The detection of endotoxin in amniotic fluid. Am J Obstet Gynecol 1987; 157: 815-819.

22. Romero R, Quintero R, Emamian M, et al. Arachidonate lypooxigenase metabolites in amniotic fluid of women with intraamniotic infection and preterm labor. Am J Obstet Gynecol 1987; 157: 1457-1660.

23. Romero R, Wu YK, Mazor M, Hobbins JC Mitchel MD. Increased amniotic fluid leucotriene $\mathrm{C} 4$ concentration in term with parturition. Am J Obstet Gynecol 1988; 159: 655-657.

24. Romero R, Brody DT, Oyarzun E, et al. Infection and labor III: Interleucin-1 a signal for the onset of parturition. Am $J$ Obstet Gynecol 1989; 160: 1117-1123.

25. Romero R, Manogue KR, Mitchel MD, et al. Infection and labor IV Cachectin-tumor necrosis factor in the amniotic fluid of women with intraamniotic infection. Am J Obstet Gynecol 1989; 161: 330-341.

26. Romero R, Wu YK, Brody DT, et al. Human Decidua: a source of Interleucin-1. Obstet Gynecol 1990; 73: 31-34.

27. Roussis P, Rosemond Rl, Glass C and Boehm F. Preterm premature Rupture of Membranes: Detection of Infection. Am J Obstet Gynecol. 1991; 165: 1099-1104.

28. Coultrip L, Lien JM, Gomez R, et al. The value of amniotic fluid interleukin- 6 determination in patients with preterm labor and intact membranes in the detection of microbial invasion of the amniotic cavity. Am J Obstet Gynecol 1994; 171: 901-911.

29. Yoon BH, Romero R, Kim JK, et al. Amniotic Fluid Interleukin-6: a sensitive test for antenatal diagnosis of acute inflammatory lesions of preterm placenta and prediction of perinatal morbidity. Am J Obstet Gynecol. 1995; 172: 960-970.

30. Gibbs RS, Romero R, Hillier SL, Eschenbach DA, Sweet RL. A Review of premature birth and subclinical infection. Am J Obstet Gynecol 1992; 166: 1515-1528. 